\title{
LA UTILIZACIÓN DE TWITTER COMO HERRAMIENTA DOCENTE: SU APLICACIÓN AL GRADO DE RELACIONES LABORALES Y EMPLEO
}

\section{Twitter as Teaching Tool: Development and Validity for the Degree of Labor Relations and Employment at Carlos III University}

Patricia Nieto Rojas

Profesora Ayudante Doctora

Universidad Carlos III de Madrid

\section{ABSTRACT}

El uso de redes sociales se ha convertido en algo generalizado, alcanzando también al mundo académico. Este artículo dará cuenta de un proyecto de innovación docente, llevado a cabo por varios profesores de la Universidad Carlos III de Madrid, consistente en la utilización de Twitter como material docente, habida cuenta que esta red social aparece como un medio altamente compatible con la educación en competencias. El proyecto surge con el convencimiento del potencial de las redes sociales como herramienta docente en varias asignaturas del área del Derecho del Trabajo aunque esta contribución se centrará en su ejecución en una asignatura obligatoria del 3. ${ }^{\text {er }}$ Curso del Grado de Relaciones Laborales y Empleo, denominada "Análisis del marco regulador de las relaciones laborales en España".

Palabras clave: innovación, Twitter, aplicación, eficacia, interactividad, Espacio Europeo de Educación Superior. 
In this article I will be discussing ways that you can use social media in the classes at university. Specifically, we have used Twitter in an innovation project at Carlos III University. Twitter is a microblogging site. Microblogging involves writing very short updates on what you are doing, your ideas, activities, to interesting sites and so on. Twitter limits updates to 140 characters, and these updates are known in the Twitter community as 'tweets'. The potential of social networks as a teaching tool in several subjects in the area of Labor Law Department is evident although this communication will focus on its implementation in a compulsory subject of the 3rd Degree of Labor Relations and Employment, called "Analysis of the regulatory framework of relations Labor in Spain».

Keywords: innovation, social media, twitter, development, validity, application, European Higher Education Area. 


\section{SUMARIO}

Sumario. 1. Introducción: las nuevas tecnologías como material docente. 2. Descripción del proyecto. 3. Términos de ejecución. 4. Su aplicación en el Grado de Relaciones Laborales y Empleo. 5. Una valoración final. 6. Bibliografía.

\section{Introducción: las nuevas tecnologías como material docente}

Las redes sociales no solo permiten publicar información, sino también compartirla, favoreciendo la comunicación entre los alumnos y el profesor al tiempo que despiertan en el estudiante su capacidad comunicativa y de trabajo en equipo, sin olvidar la posibilidad que brindan a los usuarios de mantener contacto con profesionales y expertos en la disciplina con los que los estudiantes no podrían interactuar de ninguna otra forma.

Al tiempo, la utilización de las nuevas tecnologías en el desarrollo docente no solo es una manifestación de otra forma de materializar un aprendizaje constructivista, colaborativo y por competencias, tanto dentro como fuera del aula, sino que se configura como una de las señas de identidad del Espacio Europeo de Educación Superior (EEES) a las que se suman el protagonismo del alumno frente al del profesor, la bidireccionalidad ante la unidireccionalidad y, en definitiva, otros planteamientos didácticos que consideran al estudiante un agente activo (Martínez y Raya, 2013: 142).

Conscientes de que la utilización de las redes sociales podría ser una herramienta docente útil para los estudiantes de la Universidad Carlos III de Madrid, ideamos un proyecto de innovación docente que ha sido reconocido como tal en el marco de la convocatoria competitiva impulsada por el Vicerrectorado de Estudios y Educación Digital en la 14. ${ }^{a}$ Convocatoria de Apoyo a Experiencias de Innovación Docente de la Universidad Carlos III de Madrid.

Dicho proyecto, coordinado por el profesor Daniel Pérez del Prado —@dpprado_-, parte del potencial de las redes sociales, singularmente Twitter, para la mejora docente. Entendíamos que no son pocos los blogs científicos especializados, ni escasas las instituciones, organismos, profesores y especialistas que cuentan con perfil en redes sociales (dos de los perfiles más activos de esta red social en nuestra disciplina, singularmente los profesores Rojo Torrecilla y 
Beltrán Heredia, suelen insertar otras fuentes fidedignas de información de las cuales nuestros estudiantes podrían nutrirse ${ }^{1}$ ), compartiéndose en todos ellos información de muchísima utilidad para el estudioso de las relaciones laborales. Si a ello le uníamos el generalizado uso de esta red social por nuestros estudiantes, una de las grandes virtualidades del proyecto provendría de la función de descubrimiento de nuevas fuentes a través de esta red social que, como bien es sabido, se caracteriza por permitir publicar mensajes con una extensión limitada (con un máximo de 140 caracteres). Dichos mensajes aparecerán en la pantalla (Timeline) de aquellos usuarios que sean nuestros seguidores, pero igualmente también podrán ser vistos por el resto de la comunidad si los localizan mediante el motor de búsqueda de la plataforma, de ahí la importancia de que los mensajes o tuits vinculados al proyecto contuviesen alguna clase de etiqueta que los hiciese fácilmente identificables. Son los denominados hashtags, un recurso no previsto inicialmente por los fundadores del servicio y que se basa en marcar alguna palabra clave o frase escribiéndola precedido del símbolo \#.

Estas etiquetas permiten la función discursiva, estableciendo hilos de conversación sobre un tema e incluso pudiendo entrar espontáneamente en conversaciones ajenas. En la ejecución del proyecto aquí presentado han sido utilísimas dos etiquetas, como puede comprobarse en el timeline de la autora de esta comunicación (@nietopatri).

La primera de ellas \#piduc3m e vehicular al proyecto en sí y permite visualizar de un único vistazo todos los materiales que han sido compartidos por los participantes del mismo sean los profesores implicados sean los propios estudiantes. Y la segunda, ya específica de la asignatura, es \#marcorrll y ha sido utilizada como elemento unificador de todos los materiales que tuviesen utilidad con las sesiones magistrales y para la elaboración de los supuestos prácticos realizados por los estudiantes.

El tercer mecanismo para que nuestros mensajes ganasen repercusión y cumpliesen esta finalidad divulgativa es que aquellos que los leyesen los rebotasen al resto de los usuarios. Tal acción se denomina retweet y consiste en copiar el contenido del tweet que se desee compartir y hacerlo preceder de las letras RT.

De otra parte, la propia red tiene una herramienta de organización temática y segmentada de las lecturas a través de listas en las que recopilan otros perfiles que puedan resultar de interés para el alumnado. Con ellas se ofrece a cualquier otro usuario una selección de enlaces, de modo que quien las crea ofrece su experiencia y criterio sobre determinados temas mientras que para quien las va-

1 Sin ánimo de exhaustividad se facilitó a los estudiantes implicados un listado de profesores de Derecho del Trabajo activos en esta red (v. gr. Eduardo Rojo; Antonio Baylos; Ignasi Beltrán; Borja Suárez; Jesús Cruz), también de organizaciones públicas y privadas ——AEDTSS; @ILO; @empleo_ gob- y algunas páginas webs de interés. 
lora y usa obtiene una relevante fuente de información pues puede suscribirse a ellas y disfrutar de la lectura recomendada (López y Tascón, 2013: 322).

Respecto al funcionamiento de la red en sí, hay determinados tuits, guardados como favoritos, que son considerados relevantes $y$, por tanto, no desaparecen en el rápido fluir del timeline y que pueden consultarse a fondo con posterioridad. El propio buscador de la red social (y otros generalistas como Google) contemplan una función de documentación en tiempo real sobre los temas seleccionados.

Y, finalmente, el perfil personal de los participantes en el proyecto, tanto estudiantes como profesores implicados, en tanto que función identitaria, entendíamos que debía acompañarse de una foto, una breve biografía y un campo de enlace para ampliar la información (Cuadros, 2015: 5).

Con esta sucinta exposición de la red social que pretendíamos utilizar como herramienta docente, en el siguiente epígrafe haremos una breve descripción del proyecto presentado y los objetivos que pretendíamos lograr con su puesta en marcha.

\section{Descripción del proyecto}

Partíamos de la hiperconectividad que define a la sociedad actual y de la que no se muestran ajenos los estudiantes, pues la mayoría de ellos pasan las sesiones pendientes del teléfono móvil. Es más, el hecho de que vivamos un estado de conexión permanente, incrementado a través del uso de celulares y servicios de mensajería instantánea (whatsapp, lines y similares) introduce algunas novedades en nuestra cognición, relaciones sociales, formación de la identidad, etc. Sin embargo, se ha destacado que estos dispositivos «se usan en mayor medida en humanidades que en clases de ciencias (....) lo cual parece especialmente grave cuando probablemente sea en humanidades donde más fácil, útil y necesario resulta que los alumnos adopten el papel proactivo y crítico que nos hará más libres»².

Ante esta evidencia decidimos convertir al teléfono inteligente en un aliado, tratando de facilitar el aprendizaje autónomo del alumno y una enseñanza más participativa, facilitándoles el uso de los recursos y las herramientas tan ágiles que proporciona una red social como twitter. Para ello, todos los profesores implicados en la ejecución del proyecto hemos tratado de identificar qué materia-

\footnotetext{
2 Sobre los cambios en nuestra cognición, véase: http://www.dreig.eu/caparazon/2012/12/30/ moviles-en-clase/
} 
les podían ser interesantes para nuestro alumnado, de modo que vieran que los contenidos vistos en las clases presenciales tenían un correlato en la red referida.

Además de esta finalidad, la puesta en marcha de este proyecto pretendía la consecución de estos otros objetivos, diseñados por el coordinador del mismo:

— Facilitar el flujo de información general, haciéndolo más ágil y accesible y permitiendo que los temas que se traten en clase estuviesen lo más apegados posible a la realidad. Durante su ejecución, la posibilidad de "twittear» noticias de los principales diarios o instituciones permitió que todos los alumnos pudieran tener acceso a ellas, viendo en tiempo real la conexión con lo que estudian en clase.

- Facilitar también el acceso a la información especializada o técnica que estuviese disponible en internet a través de blogs especializados, webs oficiales y perfiles de instituciones, profesores, técnicos y especialistas.

- Alterar el flujo de información, permitiendo que el mismo fuese bidireccional o multidireccional, pues no solamente se trataba de que el profesor facilitase los materiales que entendiese como más adecuados, sino de permitir también que el alumno pudiese compartir con el resto de la clase sus propias fuentes de información. A su vez este elemento permitiría observar el modo en que el alumno asimila los contenidos, obtiene fuentes de información y las analiza críticamente.

- Fomentar el análisis crítico de las fuentes. El uso colectivo de las fuentes de información no solamente fomenta la cooperación, sino que conduce a una mejor selección de las fuentes, pues el resto de los participantes podrán opinar y/o criticar la información empleada.

- Propiciar el análisis crítico de la información que podrá llevar a debates virtuales a través de las redes sociales que pudiesen complementar, en su caso, los debates en clase.

- Permitir que los dispositivos móviles, considerados para muchos el gran enemigo del aprendizaje en clase, se convirtiesen en su aliado fuera de ella e incluso en algunas ocasiones también dentro.

- Facilitar la interactuación entre el profesor y los alumnos y entre los alumnos entre sí en lo que hace al análisis y la discusión de las materias objeto de estudio a lo largo del curso.

- Fomentar el aprendizaje cooperativo y el intercambio de información y opiniones como herramientas para el crecimiento en el plano personal y académico.

- Promover la participación así como el interés por la materia objeto de estudio a través de una herramienta de sobra conocida por el alumnado pero que no suele emplearse con fines docentes. Con el uso de twitter se facilita que el alumno pueda buscar y aportar materiales sobre los temas vistos o que se van a ver en clase y facilita que pueda opinar sobre ellos. 
- Impulsar la utilización de las nuevas tecnologías como instrumento docente.

\section{Términos de ejecución}

Respecto a su ejecución, el proyecto tenía un carácter transversal, toda vez que fue puesto en marcha en varias asignaturas distintas tanto de Grado ( $\mathrm{La}$ Relación Laboral Individual» y «Derecho de la Seguridad Social» en los Grados de Derecho y de Derecho/Ciencias Políticas, «Análisis del Marco Regulador de las Relaciones Laborales en España» en el Grado de Relaciones Laborales y Empleo y "Derecho del Trabajo y la Seguridad Social» en el Grado en Administración y Dirección de Empresas) como en Posgrado («Empleo Público» en el Máster en Consultoría y Asesoramiento Jurídico-Laboral) y en algunos cursos de Humanidades de la Universidad Carlos III de Madrid.

En lo que hace al equipo de trabajo, en él hemos participado activamente cuatro profesores del área de Derecho del Trabajo, coordinados por Daniel Pérez del Prado (@dpprado), concretamente las profesoras Quintero Lima (@mariagemaquint1), Blázquez Agudo (@emblazq) y la autora de esta comunicación (@ nietopatri).

En relación a los términos de participación, el mismo era, como no podía ser de otro modo, voluntario para los estudiantes, si bien se informaba del mismo a todos ellos en la sesión inaugural de clase en la que se explicaban los términos de evaluación y principales contenidos a tratar. De este modo, al principio del cuatrimestre, los alumnos que dispusiesen de esta red social y voluntariamente quisieran participar en el proyecto, deberían seguir al profesor y respetar las reglas mínimas de funcionamiento al objeto de facilitar el debate y la intercomunicación.

Entre estas reglas, contenidas en la propia Guía Docente de cada una de las asignaturas implicadas, se incluía un anexo con las siguientes instrucciones:

- Se utilizará el hastag \#piduc3m y/o cualesquiera otros en función de la materia que se vaya tratando.

- Cualquiera los materiales "twitteados» por el profesor y/o los alumnos (en este caso, siempre que lo indicase el profesor de la asignatura) podría constituir material docente por lo que podrán ser empleados en las clases teóricas y/o prácticas y ser objeto de examen.

- En el caso de que haya alumnos que no quieran o no puedan participar en el proyecto se colgará en el espacio docente virtual de la universidad, denominado Aula Global, el TL (timeline) del profesor a los efectos de que puedan contar con los mismos materiales que sus compańeros. 
- La participación en el proyecto tiene carácter voluntario si bien se valorará a efectos de la nota práctica para aquellos alumnos/as que decidan acogerse.

Este mismo punto, vinculado a la participación activa en las clases, también podrá obtenerse por los cauces tradicionales, esto es, con la participación activa en los debates en el aula.

Los debates que pudieran suscitarse en las redes deberán respetar las reglas mínimas de cortesía y buenas formas.

\section{Su aplicación en el Grado de Relaciones Laborales y Empleo}

Habiendo desarrollado este proyecto a lo largo de este curso académico, es momento de evaluar su eficacia y valorar posibles puntos de mejora; objetivos ambos que se expondrán en el presente artículo en el que no solo se dará cumplida cuenta de cuáles han sido las potencialidades de esta herramienta como método docente, sino cuál ha sido el feedback obtenido de los alumnos, planteándose posibles respuestas a las dificultades detectadas.

El proyecto se ha llevado a cabo en una asignatura obligatoria de tercer curso del Grado de Relaciones Laborales y Empleo denominada «Análisis del marco regulador de las relaciones laborales en España», de la cual he sido responsable tanto de las sesiones prácticas como de las magistrales.

La actual coyuntura económica y sus efectos en el mercado de trabajo, unida a la tramitación parlamentaria de muchas reformas de calado en la materia objeto de la asignatura, hacían especialmente propicia la aplicación de Twitter como herramienta docente, toda vez que los medios de comunicación están realizando una cobertura muy amplia de la situación, lo que propicia un abundante caudal de información. Ahora bien, al igual que ocurría en otros proyectos de innovación docente en Ciencias Sociales que utilizaban esta plataforma (López y Tascón, 2013: 326), esta «repercusión mediática no está exenta de ruido y ha generado al menos en la sociedad española la curiosa sensación de que cualquier ciudadano de a pie se halle en condiciones de debatir sobre primas de riesgo, bonos basura, agencias de calificación, etc., a pesar de ser términos totalmente desconocidos para él hasta hace unos meses. Ya que el temario de las asignaturas de nuestra área de conocimiento para los primeros cursos de grado finaliza haciendo un repaso a la contemporaneidad, creemos que (como historiadores económicos) tenemos la obligación de hacer reflexionar a nuestros alumnos sobre los paralelismos de esta crisis con las ocurridas en los siglos XIX y XX y también, ante todo, debemos ofrecerles herramientas para que ellos mismos sean capaces de construir su propia interpretación de lo que está aconteciendo». Esta misma 
reflexión es trasladable a la materia objeto de este proyecto, habida cuenta que existe una abundantísima información sobre los debates parlamentarios y extraparlamentarios vinculados al mercado de trabajo (entre otros, conflicto de la estiba; la huelga en estructuras empresariales complejas; la emergencia de las empresas multiservicios; la degradación de las condiciones de trabajo de las camareras de piso) y considerábamos imprescindible facilitar al alumnado una lista seleccionada de recurso fiables que les permitiesen discernir entre lo importante y lo superfluo en un medio tan rápido y amplio como es Twitter.

Además, ante la pasividad detectada en ańos anteriores en la mayor parte del alumnado a la hora de tomar la palabra durante las sesiones prácticas, considerábamos imprescindible buscar algún mecanismo que contribuyera a romper esas barreras (visibles o invisibles) que habían impedido su participación activa en el aula. Para lograr este propósito, cada uno de los alumnos participantes tuvo que llevar a cabo diversas acciones en dos fases sucesivas.

En una primera, tuvieron que trabajar sobre la plataforma de microblogging la búsqueda de noticias y la publicación de contenidos propios que se relacionasen con los comentarios ya publicados por otros compañeros. Seguidamente tuvieron que identificar en Twitter las noticias más destacadas relacionadas con el tema tratado y/o emitir un tuit o retuitear un comentario sobre dicha noticia de alguien ajeno al grupo.

Con esta actividad, los estudiantes desarrollaban competencias de exploración y selección de hechos reales ocurridos recientemente, jerarquizando su importancia informativa así como habilidades relacionadas (Martínez y Raya, 2013: 144). Con la introducción de Twitter como recurso didáctico impulsábamos, al tiempo, un método de interacción entre profesor y alumno que es plenamente consistente con la educación promovida dentro del EEES y con las necesidades contempladas en las guías docentes de cada Grado verificadas por la ANECA.

Tras la Declaración de Bolonia, realizada el 19 de junio de 1999, con la que se crea el Espacio Europeo de Educación Superior, se modificó el sistema de cálculo de la docencia al medir esta en créditos europeos que no se basan solo en las horas docentes, sino en las horas de trabajo dedicadas por el estudiante a la asignatura, tanto en horas de clases magistrales, como en horas prácticas, trabajos tutelados, tiempos de estudio y exámenes.

Con esta decisión se instauró un modelo educativo basado en el aprendizaje y no en la enseñanza en la que el profesor es un mero tutor o director del proceso de aprendizaje; aspectos todos ellos que se visibilizan muy notoriamente en el proyecto que aquí estamos reseñando, toda vez que en su ejecución, el intercambio de información constante entre el profesor y los alumnos y entre los 
propios alumnos fue fundamental a la hora de aprovechar las ventajas de este método.

El profesor, en cumplimiento de esta exigencia de renovación metodológica, ha de tener presente que, según impone también el propio modelo instaurado a través de la creación del EEES, el alumno debe ser el principal protagonista de su propio aprendizaje y que él, como profesor, debe actuar como un mero guía $\mathrm{u}$ orientador de su proceso de aprendizaje. Ello implica que la labor docente del profesor y por tanto, su metodología, deben estar orientadas fundamentalmente a establecer los medios necesarios y más adecuados para conseguir que el estudiante sea capaz de actuar autónomamente en su proceso de aprendizaje, es decir, que el objetivo educativo fundamental que se debe perseguir con carácter general es que el alumno "aprenda a aprender» en un contexto de continuo e incesante cambio.

Además, el profesor, en el desarrollo de su labor docente, no debe olvidar que el aprendizaje en el marco del EEES se centra en la adquisición de determinadas competencias, entendidas estas como habilidades, destrezas, capacidades, etc., y que tal proceso no queda reducido a una concreta etapa vital de las personas, sino que debe tratarse de un aprendizaje a lo largo de toda la vida (Martín, 2008: 493).

En este contexto, la utilización de esta red social ha supuesto una herramienta metodológica encuadrada dentro de las nuevas tecnologías de información y comunicación, cuya aplicación, como se sabe, ha resultado fuertemente impulsada en el contexto del EEES por considerarla esencial para que el nuevo modelo de educación superior pueda ser implantado de manera adecuada y cumplir efectivamente sus muy importantes objetivos.

La función más importante que he de destacar del uso de Twitter como metodología docente es que los estudiantes adquieren conocimientos de la materia objeto de estudio con el uso de una red que utilizan frecuentemente pero que nunca han percibido como una herramienta docente. La utilización de Twitter supuso, al tiempo, que el profesor (en este caso, la profesora responsable de la asignatura) dejase de ser el centro de atención para convertirse en transmisora de experiencia. Ahora bien, el hecho de que el profesor dejase de ocupar el centro, no significa que su papel no revista un carácter fundamental como facilitador del aprendizaje.

La función principal del profesor es dirigir el debate, alentando el intercambio, preguntando, cuestionando los argumentos defendidos por los alumnos, advirtiendo de las posibles consecuencias no queridas derivadas de los mismos, sembrando dudas, nuevos problemas, otros interrogantes. El profesor se convierte, en definitiva, en moderador del debate, coordinando las diferentes intervenciones con el propósito de llevar al grupo hacia los objetivos inicialmente propuestos aunque todo ello se haga ahora en el marco de una discusión virtual. 
Al tiempo el uso de esta red social ha favorecido un método de aprendizaje más dinámico y estimulante, máxime porque los alumnos usan frecuentemente esta herramienta mejorando el aprendizaje mediante el análisis de materiales, notas de prensa, comentarios de opinión, enlaces a blogs jurídicos que abordan en tiempo real los contenidos vistos en clase.

Llegados a este punto, es importante recalcar que la asignatura en la que se ha llevado a cabo tiene además una evidente proyección política pues el análisis del sistema de relaciones laborales no puede entenderse solo con el análisis del Derecho positivo, sino que tiene que tener en cuenta el contexto, la ideología y los actores implicados. Este hecho despierta (o debiera despertar) un previo interés del alumnado que se enfrenta a una materia evidentemente condicionada por la situación socio-política y no a un supuesto de laboratorio.

El empleo de Twitter tiene la ventaja de que no solo es un medio familiar para el estudiante sino que se ha considerado como un método «capaz de eliminar barreras psicológicas y de generar un espacio comunicativo dificil, por no decir imposible, de alcanzar en otras circunstancias» (López y Tascón, 2013: 335). Para evidenciar la veracidad de la afirmación anterior se muestra una captura de algún debate mantenido con una alumna del Grado en Relaciones Laborales y Empleo en el que se muestran algunos de los materiales suministrados, los cuales, como se puede observar, estaban relacionados con aspectos muy candentes de la actualidad socio-política (entre otros, nulidad de los convenios de empresas multiservicios, el conflicto de la estiba y la tramitación parlamentaria del $\mathrm{RD}$ Ley o, in fine, la emergencia de nuevos actores en el marco laboral - $\mathrm{v}$. gr. la asociación "las kellys»— denunciando la degradación de las condiciones laborales de las empleadas de hotel).

Otra de las ventajas del uso de esta metodología docente es una mayor capacitación de los alumnos en el desarrollo de las habilidades comunicativas. Dado que esta técnica convierte a los alumnos en participantes activos de su propio aprendizaje ha coadyuvado al desarrollo de otras competencias pues han desarrollado habilidades de comunicación y la capacidad de argumentación jurídica en un espacio tan reducido como son los 140 caracteres que la red social Twitter establece para un tuit.

Al tiempo, su puesta en marcha ha sido útil para el manejo eficiente de las diversas fuentes de información y para familiarizarse con el trabajo de otros profesionales de la disciplina que también son activos en esta red social.

En definitiva, la utilización de las redes sociales como herramienta docente es un campo muy incipiente si bien ha demostrado efectos positivos en el plano educativo, favoreciendo la participación de los estudiantes en clase y mejorando los resultados al final del curso (Romero, 2016: 50-52). Sin embargo, en el ámbito universitario las experiencias de este tipo son, sin embargo, muy esca- 


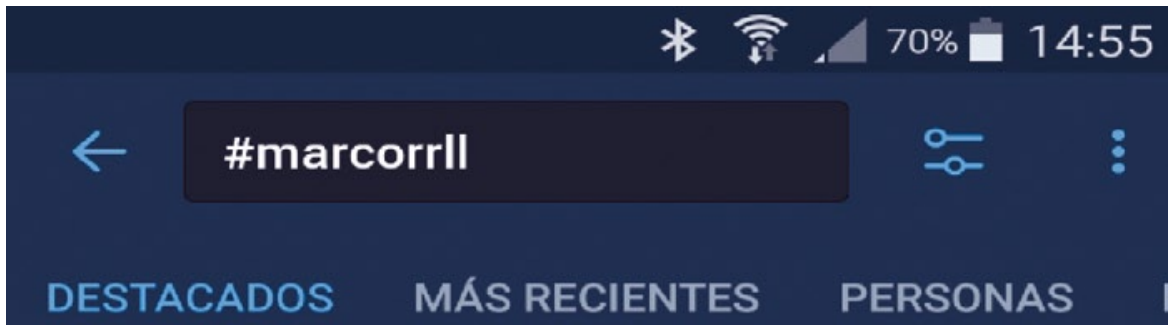

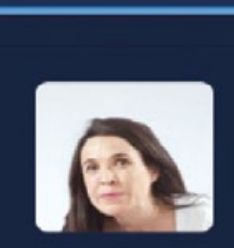

patricianieto@nietopatri.13 feb.

Efectivamente@dpprado,

utilizaremos esta sentencia en la

práctica 6 y más materiales

relacionados con conflicto C.Cola

\#piduc3m \#marcorrll

\section{Daniel Pérez@dpprado}

Posiblemente muy útil para

\#marcorrll \#piduc3m twitter.com/ erojotorrecill...
4
눈
$\bullet$
$\square$

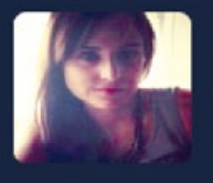

Lorena@Annerol89.13 feb.

Hoy en clase de \#marcorrll veíamos la precariedad en el trabajo que viven las Kellys \#piduc3m

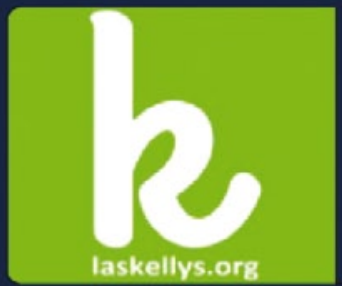

LAS KELLYS laskellys.wordp

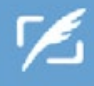


sas (Muñoz et al., 2015: 245-247; Román, 2014: 38) y prácticamente nulas en el caso particular del Derecho del Trabajo lo cual era, a juicio del coordinador del proyecto, "ciertamente paradójico, máxime si se tiene en cuenta que la materia es muy propicia, dadas sus evidentes conexiones con la realidad social, y de que si se ha desarrollado por parte de la doctrina una importante presencia en las redes sociales que, sin embargo, se ha orientado más a la investigación y la divulgación». La única excepción (al menos conocida) sería la ya referida del profesor Eduardo Rojo, catedrático de Derecho del Trabajo y de la Seguridad Social de la Universidad Autónoma de Barcelona, si bien lo ha hecho con carácter informal y a título personal, habiendo creado un espacio (http://www.eduardorojotorrecilla. es) de sinergia entre la docencia, la investigación y la divulgación digno de ser reseñado.

\section{Una valoración final}

La aprobación del proyecto de innovación docente por la Universidad Carlos III de Madrid en los términos referidos convirtió esta iniciativa en la primera experiencia docente en gozar de apoyo institucional en esta rama del Derecho, con inclusión formal en la guía docente y ejecutado entre diversos profesores del área, alcanzando a múltiples asignaturas. A la vista de lo expuesto en este artículo, creemos haber demostrado la viabilidad de utilizar las redes sociales y concretamente Twitter para mejorar el proceso de aprendizaje de los estudiantes universitarios.

Está claro que Twitter es un recurso sobre el que aún es preciso que la comunidad educativa reflexione y aporte nuevos usos y visiones. Sin embargo, queda patente, tanto en nuestra experiencia como en otras aquí referidas, su potencial como una herramienta que se adecua exitosamente a las necesidades derivadas de la puesta en marcha del Espacio Europeo de Educación Superior, sobre todo si la contraponemos a vías más tradicionales, y aunque sea pronto para valorar el éxito de la iniciativa, creemos que la ejecución del proyecto ha sido positiva. En la buena marcha del mismo han influido varios factores.

En primer lugar, el grupo en sí, y dentro de esta variable especialmente la actitud que han manifestado los estudiantes hacia el sistema. Además, al no ser un grupo especialmente numeroso (no más de 40 alumnos y asistiendo asiduamente se rebaja esta cifra hasta 30) era posible combinar la utilización de las redes sociales y la aportación de materiales mediante otras vías (correo electrónico o el propio espacio virtual habilitado por la Universidad a estos efectos).

Es obvio que la puesta en marcha de este sistema de aprendizaje dependía, en buena medida, del compromiso mostrado por parte de los alumnos 
para participar en el sistema, pues para que el debate, ahora virtual, fuese verdaderamente enriquecedor e ilustrativo eran necesarias intervenciones, ahora en Twitter, múltiples y de calidad.

En segundo lugar, en relación a la capacitación de los profesores participantes en los términos que seguidamente analizaremos. Como apuntábamos en el anterior punto, la intervención de los alumnos en el desarrollo del proyecto ha sido determinante para el éxito de esta concreta herramienta pedagógica y la autora de esta comunicación ha tenido que alentar y canalizar esta participación (ahora, virtual) al objeto de que la misma no se desviase de la cuestión nuclear y de que fuesen los propios alumnos los que fueran incorporando el uso de esta herramienta en el desarrollo docente.

Y el tercer elemento que, a mi juicio, ha sido clave para su éxito, ha sido que su ejecución se ha desarrollado en una asignatura como "Análisis del marco regulador de las relaciones laborales en España», pues la misma tiene una evidente proyección aplicativa, de ahí que haya sido utilísimo el recurso de Twitter para facilitarles materiales que mejorasen o enriqueciesen los debates que guiaban las sesiones en el aula.

Que el alumno pudiese relacionar esta específica disciplina jurídica con otras ramas del saber científico, destacadamente con la Historia y la Sociología, pero también con otras como la Economía o la Filosofía, ha resultado muy útil a los efectos de incentivar la participación en el proyecto, logrando, al tiempo, un aprendizaje más interdisciplinar; lo cual constituye otro de los rasgos principales del nuevo modelo de formación universitaria impulsado a través de la implantación del EEES.

La norma no es lo único que un egresado del Grado de Relaciones Laborales y Empleo ha de considerar para resolver un problema jurídico, sino que es importante tener en cuenta las implicaciones de las soluciones ofrecidas y valorar otras alternativas que, dentro de la legalidad, conduzcan a un mejor resultado. Es más, con el objetivo último de verificar la comprensión de los alumnos sobre los contenidos analizados, en ocasiones he introducido en el debate circunstancias distintas o implicaciones nuevas a las referidas en los supuestos prácticos que pudieran hacer variar el resultado, habiendo resultado muy formativo para esta labor los materiales proporcionados en Twitter que incluían otros problemas colaterales del debate aunque no fuesen los que de manera central se abordasen en la sesión correspondiente.

Obviamente otro elemento que ha coadyuvado al éxito del modelo es su carácter complementario y no sustitutivo de los materiales puestos a disposición del alumnado en el espacio docente virtual de la propia Universidad.

En relación a los inconvenientes de esta metodología, cabría proyectarlos en torno a dos aspectos: en primer lugar, en lo relativo a los actores implicados en 
el desarrollo de este proceso, esto es, estudiantes y profesores implicados y, en segundo lugar, en relación a la red social en sí.

Respecto al papel que debe asumir el profesor, este debe saber que los contenidos por él seleccionados condicionarán el conocimiento de la materia por el alumnado, de ahí la importancia (como, de otra parte, sucede en cualquier selección bibliografía) de la utilización de recursos fiables, objetivos y que supongan una aportación motivada al debate en cuestión.

En relación a la red en sí, en tanto que es una herramienta potentísima de información pero, al tiempo, es amplísima, ha conllevado un esfuerzo previo en la selección de los perfiles que pudiesen ser «verificados», conscientes de que nuestra selección condicionará a la del alumnado.

Sin embargo, creemos que los inconvenientes son menores que sus ventajas. La utilización de las redes sociales como herramienta docente ha servido para captar la atención del alumnado en un medio que para ellos es muy familiar al tiempo que les ha permitido conocer diferentes recursos de información que están accesibles y que suponen una fidedigna y actualizada fuente de conocimiento (sirvan nuevamente y sin ánimo de exhaustividad los activos perfiles de twitter del profesor Rojo Torrecilla o Beltrán de Heredia).

\section{Bibliografía utilizada}

Cuadros Muñoz, Roberto (2015): «Aprendizaje informal y construcción de PNL vía Twitter. Un estudio de caso». EDUTEC. Revista Electrónica de Tecnología Educativa. ISSN 1135-9250, 51, pp. 1-18, [en línea]. Disponible en: http://www.edutec.es/ revista/index.php/edutec-e/article/view/53 (Consulta: 20/06/2017).

LARA, Tiscar (2011): «Competencia digital, nuevos medios, nuevos lenguajes, nuevos hablantes. Twitter y sus funciones comunicativas», Lenguaje y Textos, 34, pp. 39-46 [en línea]. Disponible en: http://tiscar.com/2012/03/11/Twitter-y-sus-funcionescomunicativas/ (Consulta: 20/06/2017).

López Zapico, Misael Arturo; Tascón Fernández, Julio (2013): «El uso de Twitter como herramienta para la enseñanza universitaria en el caso de las ciencias sociales. Un estudio de caso desde la historia económica». Teoría de la Educación. Universidad de Salamanca, 14 (1), [en línea]. Disponible en: http://www.redalyc.org/articulo. oa?id=201028055014 (Consulta: 10/07/2017).

Martín Hernández, María Luisa (2008): «Aprender Derecho del Trabajo a través del cine», Revista Aranzadi de Derecho de Deporte y Entretenimiento, vol. 22, pp. 489-498.

Martínez-Rodrigo, Estrella; Raya González, Pura: (2013). El microblogging en el proceso de enseńanza-aprendizaje. Una experiencia académica con Twitter. Historia y Comunicación Social, 18, N.o Especial Noviembre, pp. 139-149, [en línea]. Disponible en: http://revistas.ucm.es/index.php/HICS/article/view/44232 (Consulta: 20/06/2017). 
Muñoz González, Juan Manuel (et al.) (2015): «¿Son adictos a las redes sociales los estudiantes universitarios?», Revista Complutense de Educación, 25, pp. 233-251, [en línea]. Disponible en: http://revistas.ucm.es/index.php/RCED/article/view/46659/45941 (Consulta: 20/06/2017).

Román Graván, Pedro; Martín Gutiérrez, Ángela (2014): «Las redes sociales como herramientas para la adquisición de competencias en la universidad: los códigos QR a través de Facebook", International Journal of Educational Technology in Higher Education, 11, pp. 27-42, [en línea]. Disponible en: http://rusc.uoc.edu/rusc/es/index.php/ rusc/article/view/v11n2-roman-martin.html (Consulta: 10/07/2017).

Romero López, Sebastián (2016): «Redes Sociales de Refuerzo Positivo: Interacciones Sociales Online para Medir, Analizar y Mejorar Resultados Académicos», Universidad Castilla-La Mancha [en línea]. Disponible en: https://ruidera.uclm.es/xmlui/ handle/10578/10931 (Consulta: 10/6/2017). 